\title{
A pharmaco-epidemiological analysis of factors associated with antimicrobial consumption level in turkey broiler flocks
}

\author{
Claire CHAUVIN ${ }^{\mathrm{a} *}$, Isabelle BoUvAREL ${ }^{\mathrm{b}}$, Pierre-Alexandre BELCIL ${ }^{\mathrm{a}}$, \\ Jean-Pierre ORAND ${ }^{\mathrm{c}}$, Didier GUILLEMOT ${ }^{\mathrm{d}}$, Pascal SANDERS ${ }^{\mathrm{e}}$ \\ a AFSSA, French Agency for Food Safety, Pig and Poultry Research Laboratory, \\ Epidemiology and Quality Assurance Unit, Zoopôle, BP 53, 22440 Ploufragan, France \\ ${ }^{\mathrm{b}}$ ITAVI, Technical Institute for Poultry Production, 28 rue du Rocher, 75008 Paris, France \\ ${ }^{\mathrm{c}}$ French Ministry of Agriculture, Food Fisheries and Rural Affairs, 251 rue de Vaugirard, \\ 75732 Paris Cedex 15, France \\ ${ }^{\mathrm{d}}$ Institut Pasteur, Resource Center for Biostatistics, Epidemiology and Pharmacoepidemiology \\ applied to Infectious Diseases and INSERM UMR 657, 25-28 rue du Dr Roux, 75015 Paris, France \\ ${ }^{\mathrm{e}}$ AFSSA, French Agency for Food Safety, Laboratory for the Research and Study of Veterinary \\ Medicinal Products and Disinfectants, La Haute Marche Javené, 35302 Fougères, France
}

(Received 26 May 2004; accepted 12 October 2004)

\begin{abstract}
An on-farm pharmaco-epidemiological survey of 246 turkey broiler flocks from 131 farms was carried out to assess the homogeneity of antimicrobial use between flocks on the same farm and to explore the possible relationships between farm and farmer characteristics and the level of antimicrobial use. The antimicrobial use in each flock was quantified by an invoice study, expressed as the number of national animal daily doses (ADD)/turkey broiler and characterised as "high", "medium" or "low" according to the tertiles of the resulting distribution. Antimicrobial use was then correlated with variables collected from the farmer by means of an alternating logistic regression method which calculates the pairwise odds ratio (PWOR) for within-farm clustering. Two independent models were fitted: (1) "low" versus "medium" + "high" antimicrobial consumption and (2) "high" versus "medium" + "low" antimicrobial consumption. PWOR from the null models were significant $(P<0.005)$, but only remained significant in the first final model $(P=0.002)$. Four explanatory variables were retained for both models. Prophylactic antimicrobial administration and veterinarian antimicrobial prescription attaining the farm technical staff's expectation were associated with a higher antimicrobial consumption level. Administration of competitive exclusion flora and compliance with biosecurity rules of changing clothes and shoes before entering the facilities, were associated with a lower antimicrobial consumption level. In the first model, the number of full-time jobs devoted to the turkey production unit (1 versus more than 1) was also found to be associated with the antimicrobial consumption level. The study tends to confirm the feasibility of the adopted approach to quantify antimicrobial use and to determine the factors likely to influence antimicrobial consumption.
\end{abstract}

antimicrobial / pharmaco-epidemiology / alternating logistic regression (ALR) / turkey broiler / animal daily dose (ADD)

\footnotetext{
* Corresponding author: c.chauvin@ploufragan.afssa.fr
} 


\section{INTRODUCTION}

Antimicrobial use in food-producing animals has become a major concern in veterinary public health, especially in relation to the risk of emergence and dissemination of resistant bacteria. The investigation and monitoring of antimicrobial use in animal husbandry, with the surveillance of the quantities administered together with the patterns of use, has therefore been widely recommended [22]. Improving our knowledge of these characteristics should be useful in designing, implementing and promoting judicious use of such compounds. Pharmacoepidemiological studies targeted to identify the factors influencing antimicrobial consumption will be of considerable value to the professionals. In the context of veterinary public health, the key points identified to be related to antimicrobial use could lead to modification of the exposure of animals to antimicrobials in order to reduce the risk of bacterial resistance. Farmers would be all the more involved and convinced of the value of decreasing antimicrobial exposure, since they are also concerned by the cost of antimicrobials. A reduction in antimicrobial expenditure, resulting from decreased use, would be of particular interest, due to the marked influence that antimicrobials have on total health maintenance expenditure, which, in France, represented the second variable production cost in turkey broiler production in 2001 [24].

Moreover, considerable variability has been observed in these antimicrobial expenditures [24]. However few studies have been carried out on the relationships between farm and farmer characteristics and antimicrobial use. These have either focused on the use of particular antimicrobials [12] or were limited to a particular relationship hypothesis [17] and only a few have been based on the quantification of antimicrobial use mainly according to frequency of use $[23,32]$. None of these studies concerned poultry production and the few relationships identified between antimicrobial consumption and various covariates cannot be extrapolated to turkey broiler production. The homogeneity of consumption between successive batches of animals on the same farm, which would suggest that certain farm or farmer characteristics influence antimicrobial consumption, has never been investigated.

Thus, the object of this study was to conduct an observational on-farm investigation in turkey broiler flocks, to assess the homogeneity of antimicrobial use between flocks on the same farm and to identify possible relationships between the level of antimicrobial use and farm, farmer and flock characteristics.

\section{MATERIALS AND METHODS}

\subsection{Study sample}

This study was conducted in eleven departments of the three predominant regions of French turkey production (Centre, Bretagne, Pays de la Loire), which represent $46.2 \%$, $20.7 \%$ and $7.1 \%$ of the total turkey production, respectively [4].

Farms were randomly selected, with a five percent sampling rate, from the departmental farming registers. The farms included in this study were specialised turkey farms with the exclusion of rare, more specialised productions, such as "light turkey" farms. Farmers were contacted by telephone to explain the objective of the survey and to obtain their agreement to participate. The farmers who declined to take part could not be characterised and thus compared with the farmers included in the study, so the final sample of flocks was compared retrospectively to a broad data base from the same geographical area [2] on the average age and weight at slaughter, density, average daily weight gain and mortality rate.

\subsection{Data collection}

An appointment was made at the first telephone call to perform data collection on the farm. The farmer was informed about 
the data collection procedure, based on a personal interview and an invoice study. For this purpose, he was asked to prepare all invoices related to the flocks reared during the last twelve months (between the 1st of April 2000 and the 30th of March 2001) and to make them available to the investigator on the day of the visit. The farms included in the study were visited between March and July 2001.

The interviews and invoice studies were carried out by ten experienced investigators who had received suitable training during a run-in period and were affiliated to professional agricultural bodies in the region. First, structured questionnaires (Tab. I) were put to the farmers to obtain information about farm characteristics, the farmer's attitudes and habits in relation to health management and the technical performance of the flocks. The questionnaire about farm characteristics had already been in routine use for several years. The questionnaire related to health management had been pilot-tested by interviewers from each professional agricultural body, on a separate random sample of five flocks. Only minor changes, based on these results and feedback, needed to be made to this questionnaire.

Secondly, antimicrobial use was investigated from a survey of medication purchases. All invoices were thoroughly analysed. When the purchase of a veterinary medicinal product containing an antimicrobial was indicated, the trade names, presentation characteristics (weight or volume, active compound concentration, etc.) and quantities (number of package units purchased) were recorded. Purchases were attributed to a flock according to the date of invoicing and the farmer's indications.

\subsection{Outcome variable definition}

For each commercial presentation of antimicrobial, the number of commercial units purchased was converted into the weight of the corresponding active substances, according to the nominal composition of the veterinary medicines purchased [3]. The quantities of active substances were all expressed in terms of the same standard chemical form according to the official chemical conversion coefficients. The number of animal daily doses (ADD) purchased was then calculated for each antimicrobial active substance. The ADD is a measurement unit of drug use developed as a counterpart to the defined daily dose (DDD) [16] but corresponding to national practices or recommendations. The DDD was defined in human medicine as the theoretical average maintenance dose per day for the drug used in its main indication in adults [20]. Similarly the $\mathrm{DDD}_{\text {animal }}$ would be the assumed average dose per day for a drug used for its main indication in animals. The ADD were used in the present study since no DDD turkey $_{\text {has }}$ as yet been officially defined in turkey production and the use of the term DDD would be limited to daily doses corresponding to the international standards for drug use measurement. These corresponded to the recommended dosage approved in France for each antimicrobial [3] to treat during one day one turkey broiler weighing three kilograms. This weight was the average turkey broiler weight at treatment, based on a large number of treatments $(N=1160)$ recorded by an antimicrobial use surveillance network [11]. One ADD, therefore, corresponded to approximately one treatment day during the average higher risk period for antimicrobial administration (corresponding to a three kilograms live weight). The weight of each antimicrobial purchased was divided by its corresponding daily dose (the corresponding ADD for each antimicrobial are available on request from the authors). Finally the total number of ADD purchased by the farmer was divided by the number of turkey broilers reared in the corresponding flock.

In order to characterise and qualify flock antimicrobial consumption in a semi-quantitative way, a three-level ordinal variable was defined according to the tertiles of the 
Table I. Summary of factors included in the questionnaires used to analyse factors associated with antimicrobial use in turkey broiler production (131 farms, 246 flocks, France, 2001).

\section{General items related to the farm}

\section{. Farm characteristics}

- Farm staff characteristics (number, age, experience, education)

- Facility characteristics (number, size, age, equipment)

- Crop land surface

- Other animal production

- Location, farm density

\section{. Biosecurity}

- Production specifications

- Access to facilities and surroundings

- Working procedures

- Hygiene procedures (cleaning and disinfecting, dead animals disposal, ...)

- Control of wildlife (rodents, insects)

\section{Health management practices}

- Veterinary medicine provider characteristics

- Treatment decision process (autopsy, analysis, antibiograms, ...)

- Treatment administration equipment (pump, etc.) and procedure

- Systematic autopsy, analysis

- Water treatment and analyses

- Farmer perception (level of use, perceived risks, etc.)

\section{Flock performances and history}

\section{. Technical and economic results ${ }^{\mathrm{a}}$}

- Production type

- Stocking and slaughtering dates

- Density

- Feed type

- Mortality

- Slaughterhouse condemnation rate

- Economic results

\section{. Treatments received}

- Vaccination scheme $\mathrm{b}^{\mathrm{b}}$

- Prophylactic antimicrobial treatment received (age, nature, reason, etc. $)^{\mathrm{b}}$

- Medications administered (vitamins, acids, competitive exclusion flora, etc. $)^{b}$

\footnotetext{
a These elements were also collected or confirmed from on-farm records, slaughterhouse and production organisation documents, feedstuff delivery orders.

b The farmers' answers were confirmed or completed by the invoice study.
}

antimicrobial consumption distribution (in ADD/turkey broiler), estimated from the whole sample of flocks. The flock antimicrobial consumption was thus classified as "low", "medium" or "high".

\subsection{Statistical analysis}

Two binary outcomes were defined, corresponding to two different cumulative models: (1) "low" antimicrobial consumption 
versus "medium" + "high", and (2) "high" antimicrobial consumption versus "medium" + "low". They were subjected to two independent logistic regression models designed to identify those characteristics of the farm, farmer and flock which were associated with "low" or "high" antimicrobial consumption levels, respectively.

The farm cluster was taken into account by subjecting the data to an alternating logistic regression (ALR) in order to estimate the pairwise odds ratios (PWOR) for the within farm association, while simultaneously regressing the binary outcome on covariates [8]. The PWOR reflects how strongly a given event (e.g. "high" level antimicrobial consumption) occurs in clusters (i.e. within farms ). It reflects the odds of the event for an individual in a particular cluster given that another individual from that same cluster experienced the event, relative to the odds if that individual did not experience the event. For the binary variable $\mathrm{Y}_{\mathrm{ij}}$ denoting the antimicrobial consumption level for the jth flock in the ith farm, the log PWOR model is written as the following equation: $\log \left(\operatorname{PWOR}\left(\mathrm{Y}_{\mathrm{ij}}, \mathrm{Y}_{\mathrm{ij}}\right)\right)=\alpha . \mathrm{Z}_{\mathrm{ijj}}$, where $\alpha$ represents a $q \times 1$ vector of regression parameters and $\mathrm{Z}_{\mathrm{ijj}}$, represents a fixed specified vector of coefficients. At the same time, logistic regression was used to control covariates, according to the following standard formula: $\operatorname{Logit}\left(\mathrm{P}\left(\mathrm{Y}_{\mathrm{ij}}=1\right)\right)=\beta_{0}+\beta_{1} \mathrm{X}_{1 \mathrm{ij}}+$ $\ldots+\beta_{n} X_{n i j}$, where $Y_{i j}$ is the outcome for the jth flock in the ith farm and the $X_{i j}$ are the covariates associated with that outcome. $\alpha$ and $\beta$ were estimated by iterative recalculation of the PWOR and logistic regression on the covariates $[8,18]$.

Firstly, the associations between antimicrobial consumption and the variables collected in the questionnaire were checked in a univariable step. Explanatory variables were categorically coded, with more than $10 \%$ of the sample size in each class made. Variables associated with $P<0.25$ were retained. Any strong colinearity $(P<0.05)$ between explanatory variables was checked and the variable most strongly associated with the outcome variable was retained. The retained variables were then introduced into a multivariable logistic model fitted with a backward selection procedure. All variables with $P<0.05$ were kept in the final two models. Null models without any explanatory variable were also fitted to estimate the initial PWOR. ALR was applied using the GENMOD procedure of the SAS software (SAS Institute, Inc.).

\section{RESULTS}

\subsection{Flock sample}

Antimicrobial consumption was investigated in 246 flocks from 131 visited farms. Fewer than $10 \%$ of the contacted farmers refused a visit, mainly due to lack of time. The flock sample profile was similar to that of the reference database. The flocks studied were stocked in the facilities between the 3rd of February 2000 and the 1st of February 2001. Each flock contained 10737 turkey broilers on average (standard deviation $=4817$ ) housed in facilities of $1364 \mathrm{~m}^{2}$ on average (standard deviation $=630$ ). . During the twelve-month test period, two flocks of turkey broilers had been reared in $83 \%$ of the farms visited (109/131), three flocks were studied on three farms and a single flock on the remaining 19 farms. These latter farms did not contribute directly to the PWOR because they could not be paired with another flock on the same farm.

Each tertile of the antimicrobial consumption distribution comprised eighty-two flocks. The first class corresponded to "low" antimicrobial use i.e. from 0 to less than $5 \mathrm{ADD} /$ turkey broiler, and the second and third classes corresponded respectively to "medium" use i.e. from 5 to $15 \mathrm{ADD} /$ turkey broiler and "high" antimicrobial consumption, corresponding to more than $15 \mathrm{ADD} /$ turkey broiler.

When two successive flocks on the same farm were examined (Tab. II), they were situated in the same category in $53 \%$ of the 
Table II. Comparison of antimicrobial consumption category between two successive flocks from the same farm (227 flocks, 115 pairs, 112 farms, France, 2001).

\begin{tabular}{lcc}
\hline \multicolumn{2}{c}{ Antimicrobial consumption category } & $\begin{array}{c}\text { Number } \\
\text { of pairs }\end{array}$ \\
\cline { 1 - 2 } $\begin{array}{l}\text { First flock } \\
\text { in the pair }\end{array}$ & $\begin{array}{c}\text { Second flock } \\
\text { in the pair }\end{array}$ & \\
\hline low & low & 28 \\
low & medium & 10 \\
low & high & 2 \\
medium & low & 9 \\
medium & medium & 14 \\
medium & high & 18 \\
high & low & 4 \\
high & medium & 11 \\
high & high & 19 \\
\hline
\end{tabular}

cases (61/115), in adjacent categories in $42 \%$ of the cases (48/115), and in the two extreme categories in 6 cases $(5 \%)$. The level of antimicrobial consumption increased between two successive flocks in $26 \%$ of the cases (30/115) and decreased in 21\% (24/115).

\subsection{Antimicrobial consumption analysis}

The initial PWOR was $11.4(P<0.0001$; $\left.\mathrm{CI}_{95 \%}=4.5,28.8\right)$ in the first null model ("low" versus "medium" + "high" antimicrobial consumption) and $3.6(P=0.002$; $\left.\mathrm{CI}_{95 \%}=1.6,8.0\right)$ in the second null model ("high" versus "low" + "medium" antimicrobial consumption).

The variables retained after the univariable step are presented in Table III. No relationship was found between the technical and economic performances of the flocks and the level of antimicrobial consumption.

The final models obtained $(P<0.05)$ are presented in Table IV. Four variables were common to both models: prophylactic antimicrobial administration, veterinarian antimicrobial prescription attaining the farm technical staff's expectation, administration of competitive exclusion flora and compliance with the biosecurity rules of changing clothes and shoes before entering the facilities. In the first model, the PWOR and a fifth variable i.e. the number of full-time jobs devoted to the turkey production unit (1 versus more than 1 ), were significant.

\section{DISCUSSION}

The antimicrobial consumption of turkey broiler flocks was studied in a large number of farms which had been randomly selected from departmental databases. Very few farmer refusals were recorded. This could be attributed to the five months inclusion period which offered sufficient opportunities for fixing appointments. However it was more likely due to the interest of both farmers and professionals in a study that dealt with animal health and antimicrobial consumption, a particularly important topic in 2001 following the ban of antimicrobial growth promoters. The technical characteristics of the flocks included in this study were similar to the mean values calculated on a larger sample at a regional level. It is therefore reasonable to assume that data quality was not affected by selection bias. In addition the questionnaire had been pilot tested and the investigators were experienced and suitably trained which would minimise investigator bias.

The antimicrobial consumption of each flock was determined from the farmer's invoice records. Due to packaging constraints, the quantities purchased could be greater than those actually required to treat the animals so this method can be considered to overestimate antimicrobial consumption. We nevertheless considered that invoice records were the best way to collect this information. All farmers keep all their invoices for accounting purposes. An alternative method, based on on-farm records, could have been marred by partial and/or imprecise recording of treatments. Data collection from veterinarians (antimicrobials in France have to 
Table III. Definition and distribution of explanatory variables selected after univariable analysis of antimicrobial consumption of turkey broiler flocks, expressed in ADD/turkey broiler and categorised in a three level variable ("low", "medium" and "high", corresponding to the tertiles of the distribution) (131 turkey farms, 246 turkey broiler flocks, France, 2001).

\begin{tabular}{|c|c|c|c|c|c|}
\hline \multirow[t]{2}{*}{ Variables } & \multirow{2}{*}{$\begin{array}{c}\% \text { of } \\
\text { flocks }\end{array}$} & \multicolumn{2}{|c|}{ Distribution per level } & \multicolumn{2}{|c|}{$P^{\mathrm{a}}$} \\
\hline & & $\begin{array}{l}\% \text { in "low" } \\
\text { categoryc }\end{array}$ & $\begin{array}{l}\text { \% in "high" } \\
\text { categoryc }\end{array}$ & $\begin{array}{c}\text { First } \\
\text { model }^{d}\end{array}$ & $\begin{array}{l}\text { Second } \\
\text { model }^{\mathrm{e}}\end{array}$ \\
\hline Farmer has received poultry production education & & & & 0.25 & - \\
\hline Yes & 32.9 & 28.4 & 34.6 & & \\
\hline No & 67.1 & 35.8 & 32.7 & & \\
\hline Number of full-time jobs devoted to turkey broiler production ${ }^{\mathrm{fg}}$ & & & & 0.03 & 0.19 \\
\hline One & 87.4 & 34.9 & 33.0 & & \\
\hline . More than one & 12.6 & 22.6 & 35.5 & & \\
\hline Crop land surface $\mathrm{fg}^{\mathrm{fg}}$ & & & & 0.05 & 0.05 \\
\hline$<50$ ha & 63.4 & 30.1 & 38.5 & & \\
\hline.$\geq 50$ ha & 36.6 & 38.9 & 24.4 & & \\
\hline Number of facilities on the farm & & & & - & 0.09 \\
\hline One & 76.4 & 31.4 & 37.2 & & \\
\hline . More than one & 23.6 & 39.7 & 20.7 & & \\
\hline Age of facilities ${ }^{\mathrm{fg}}$ & & & & 0.05 & 0.04 \\
\hline$<10$ years & 48.4 & 41.2 & 26.9 & & \\
\hline.$\geq 10$ years & 51.6 & 26.0 & 39.4 & & \\
\hline Concrete floor in the facilities & & & & 0.22 & 0.16 \\
\hline . Yes & 11.4 & 50.0 & 17.9 & & \\
\hline . No & 88.6 & 31.2 & 35.3 & & \\
\hline Double disinfection of the facilities before stocking a new flock ${ }^{g}$ & & & & - & 0.24 \\
\hline Yes & 56.9 & 35.7 & 30.0 & & \\
\hline No & 43.1 & 30.2 & 37.7 & & \\
\hline Systematic autopsies (necropsies) during rearing $\mathrm{g}$ & & & & - & 0.03 \\
\hline Yes & 30.9 & 30.3 & 22.4 & & \\
\hline No & 69.1 & 34.7 & 38.2 & & \\
\hline Necropsy of day-old animals & & & & - & 0.12 \\
\hline Systematic & 33.3 & 28.0 & 30.5 & & \\
\hline In case of problem & 23.6 & 37.9 & 12.1 & & \\
\hline Never & 43.1 & 34.9 & 47.2 & & \\
\hline Clothes and shoes changed on entering the facilities ${ }^{\text {fg }}$ & & & & 0.06 & 0.03 \\
\hline Yes & 56.5 & 39.6 & 26.6 & & \\
\hline . No & 43.5 & 25.2 & 42.1 & & \\
\hline Hand washing before entering the facilities & & & & 0.05 & 0.08 \\
\hline Yes & 54.9 & 40.0 & 27.4 & & \\
\hline No & 45.1 & 25.2 & 40.5 & & \\
\hline Prophylactic antimicrobial treatment ${ }^{\mathrm{fg}}$ & & & & 0.02 & 0.002 \\
\hline Yes & 25.6 & 17.5 & 50.8 & & \\
\hline . No & 74.4 & 38.8 & 27.3 & & \\
\hline Competitive exclusion flora administration ${ }^{\text {fg }}$ & & & & 0.05 & 0.04 \\
\hline Yes & 11.8 & 58.6 & 10.3 & & \\
\hline . No & 88.2 & 30.0 & 36.4 & & \\
\hline Veterinarian antimicrobial prescriptions ${ }^{\mathrm{fg}}$ & & & & 0.06 & 0.05 \\
\hline Came up to the farm technical staff expectation & 57.3 & 24.1 & 40.4 & & \\
\hline Were not particularly expected & 42.7 & 45.7 & 23.8 & & \\
\hline
\end{tabular}

a Probability for the variable introduced in an alternating logistic regression model with within-farm PWOR estimation.

b Marginal column percentage (i.e. percentage of the 246 flocks in each level of the explanatory variable).

c Row percentages (i.e. percentage of flocks, corresponding to an explanatory variable modality, in the "low" or

"high" level of antimicrobial consumption).

dFirst model consisted of the comparison of "low" antimicrobial consumption versus "medium" and "high" levels.

e Second model consisted of the comparison of "high" versus "medium" and "low" levels.

${ }^{\mathrm{f}}$ Variables introduced into the first multivariable model.

$\mathrm{g}$ Variables introduced into the second multivariable model. 
Table IV. Final cumulative ALR models for antimicrobial consumption in ADD/turkey broiler, categorised in three levels: "low", "medium" and "high" (131 farms, 246 turkey broiler flocks, France, 2001).

Variables

OR

$95 \% \mathrm{CI}$

$\mathrm{P}$

First model: "low" antimicrobial consumption versus "medium" and "high" antimicrobial consumption

Number of full-time jobs devoted to turkey broiler production

One

$5.3 \quad 1.7-16.5 \quad 0.004$

. More than one

Ref. $^{\text {b }}$

Clothes and shoes changed on entering the facilities

. Yes

$1.2-6.2$

0.02

. No

Ref

Veterinarian antimicrobial prescriptions

. Came up to the farm technical staff expectations

0.4

$0.2-0.8$

0.01

. Were not particularly expected

Ref.

Competitive exclusion flora administration

$\begin{array}{lccc}\text {. Yes } & 3.9 & 1.4-11.1 & 0.01 \\ \text {. No } & \text { Ref. } & - & \end{array}$

Prophylactic antimicrobial treatment

. Yes

0.3

$0.1-0.8$

0.02

. No

Ref.

Farm pair-wise odds ratio
5.9
$1.9-18.1$
0.002

Second model: "high" antimicrobial consumption versus "medium" and "low" antimicrobial consumption " Clothes and shoes changed on entering the facilities
. Yes
0.4
$0.2-0.7$
0.006
. No
Ref.

Veterinarian antimicrobial prescriptions

. Came up to the farm technical staff expectations

$1.2-5.2$

0.02

.Were not particularly expected

Ref

Competitive exclusion flora administration

. Yes

$0.1 \quad 0.02-0.7$

0.02

. No

Ref.

Prophylactic antimicrobial treatment

$$
\text { . Yes }
$$

$1.6-7.4$

0.001

Ref.

Farm pair-wise odds ratio

$1.7 \quad 0.6-4.8$

a Intercept: -2.0 .

b Reference level.

c Intercept: -1.1 . 
be prescribed by a veterinarian) would have come up against the fact that more than one veterinarian may prescribe antimicrobials to the same farm. Invoices also provided valuable and detailed information about the names of the commercial products, the presentations and the quantities acquired. Moreover, the quantities of active compounds purchased could be precisely calculated from the product composition data [3], and the invoices.

An ADD measurement unit was used to express and quantify the total purchases of antimicrobials. This is a national unit, but similar to the international DDD, and allows standardisation of medication consumption [9], in this case antimicrobials, by correcting for differences in dosages. Its application has therefore been recommended in studies of antimicrobial use [22]. The ADD measurement unit - often called DDD - has occasionally been used to quantify national antimicrobial consumption in poultry [5, 14] and other species [5, 13], but has only been used in pigs to measure antimicrobial consumption on farms [19]. The ADD defined for the purposes of this study were based on French criteria and may differ from other national ADD or international DDD. Their definition was based on a reference weight which would not reflect any heterogeneity in animal weight at the time of treatment. The under- or over-estimation of antimicrobial consumption considered as a "number of days of treatment received" was limited by taking the average weight at treatment in French turkey broiler production as the reference weight. This weight may be different in other countries or periods.

The number of ADD/animal was considered as a measure of a potential selected pressure imposed on flock bacterial flora, as evoked by Jensen et al. [16] and not as a measure of treatments. A treatment administered in the earliest stages of the rearing period would contribute less to the total consumption than a treatment administered at later stages. This has already been pointed out in estimations of the use of antibiotics in human medicine in both children and adults [9]. Similarly, the contribution of a treatment to the total consumption will depend on whether the administered dosage is greater or smaller than the recommended dosage used to define the ADD, and on the treatment length. However the administered dosages, treatment length and age at treatment seem to be less variable in poultry production [11] than in pig production [10].

In the present study, the total antimicrobial consumption per turkey broiler (measured in number of ADD/turkey broiler) was not analysed as a continuous variable. It was subsequently dichotomised in order to determine the characteristics of the extreme tertiles - "high" or "low" level of antimicrobial consumption - for more detailed analysis of distinct antimicrobial consumption profiles. Categorisation of the number of ADD/turkey broiler was based on the tertiles of the distribution since no natural or official cut-off value could be determined from the distribution or from the literature.

The statistical method used in the analysis was also original. The recently introduced ALR method [8] allows the assessment of the similarity between flocks on the same farm and estimation of the effects of covariates on the flock's antimicrobial consumption. ALR are increasingly used in social epidemiology to determine a contextual influence [27-29], and the resulting PWOR can readily be interpreted to quantify the magnitude of within-cluster association. Flocks reared on the same farm cannot be considered to be independent, since they are reared in the same environment (facilities, geographic location, etc.) by the same farm personnel. An analysis of the homogeneity of antimicrobial consumption between flocks, under such conditions, was therefore important. The PWOR in the null models were highly significant, demonstrating a high similarity in antimicrobial consumption between flocks on the same farm. These PWOR decreased markedly when adjustments were made for farm and farmer 
characteristics (most of which were collected at the farm level). The within-farm PWOR remained significant in the first model, suggesting that clustering of "low" antimicrobial consumption among flocks in a farm was not entirely explained by the retained covariates. In contrast, the withinfarm PWOR became non-significant in the second model, suggesting that the clustering of "high" antimicrobial consumption was largely due to the covariates. These results also suggest that a "high" level of antimicrobial consumption is probably due to other parameters independent of the farm and the farmer. However, the same explanatory variables were retained in both models. An additional fifth variable was only retained in the first model. Moreover, the four combined variables presented relatively constant coefficients according to the dichotomisation (i.e. the estimated $\beta$ and $\mathrm{CI}_{95 \%}$ were similar for the "low" versus "medium" + "high" and "low" + "medium" versus "high" comparisons). This suggests that the observed effect of the variables is the same regardless of the cut-off value considered [1].

The observed protective effect of administration of competitive exclusion flora to the turkeys can be related to the reported effect of competitive exclusion flora on the equilibrium and regulation of the digestive tract flora and their consequent role and use in the prevention of digestive tract disorders [25]. When the data was collected in 2001, there had been major modifications in poultry feedstuffs, due to the ban of growth promoters and meat and bone meals in 1999 and 2000, respectively. This situation may have led to an increase of digestive tract disorders $[14,26,33]$. In this particular context, competitive exclusion flora would often have been used and would have been all the more efficient in the present study.

Another health management practice was included in the models with an adjustment made for the prophylactic use of antimicrobials. The administration of a prophylactic antimicrobial treatment was significantly associated with a higher level of antimicrobial consumption. These prophylactic treatments were administered during "high-risk" periods when the flock was known to be more susceptible to infectious diseases (based on previous experience or observed infections in an adjacent facility, for example). Their use was especially frequent in 2001 when the survey was performed, due to the appearance and fear of necrotic enteritis following the ban on growth-promoting antimicrobials $[14,33]$.

Antimicrobial consumption was likely to be higher when veterinarian antimicrobial prescriptions were more likely to meet the farm technical staff expectations, than when antimicrobial prescriptions were not particularly expected. Several hypotheses can be put forward to explain this observation. Some farmers may be used to encountering infectious diseases and being able to anticipate and recognise the therapeutic needs of their animals. The use of antimicrobials might therefore be considered as a marker of a particularly affected sanitary situation. It might also reflect the farmer's knowledge of his practitioner's habits. By comparison with observations previously made in human medicine concerning the prescription of antimicrobials by paediatricians [21] and general practitioners [7], it might also reflect a possible influence of the farmers expectations on veterinarian prescriptions. An interaction between the farmer and veterinarian has already been reported to influence the pattern of antimicrobial use on Michigan dairy farms [17] while in Denmark the farmer was suspected to directly influence antimicrobial use in pig production [6]. In human medicine, practitioners have been found to be more likely to prescribe an antimicrobial when parents or patient are expecting one [7, 21]. Like such parents, expectation on the part of the farmers may convey the attention they pay to their animals and their hopes for their recovery. However a distinction must be made between these two situations: antimicrobials are often reimbursed in human medicine but represent a considerable 
expense in turkey broiler production [24], which may limit any overuse of an antimicrobial due to the farmers expectations (the appropriateness of the prescription (possible cases of over-use/misuse) was not assessed in the present study). Further studies are required to explore the potential influence of farmer expectation on antimicrobial prescription and consumption.

The application of good rearing practices and basic rules of biosecurity, i.e. changing clothes and shoes before entering a facility, was found to be associated with lower antimicrobial consumption. These variables might be a reflection of the application of good farming practices and biosecurity rules by the farmer. Biosecurity measures may contribute to a lower use of antimicrobials by their protective effect against the introduction and spread of infectious agents [31].

The only factor specific to the first model and associated with the lowest level of antimicrobial consumption was the number of full-time jobs devoted to turkey broiler production. This could be related to the size of the turkey broiler production unit and these two variables were correlated in the present study (data not shown). A large herd size has already proved to be a risk factor for mortality and infectious diseases in many species, including poultry $[15,30]$.

The relationships assessed in the present study between the level of antimicrobial consumption and farm, farmer and flock characteristics are fairly different from those reported in the limited number of published studies [23, 32]. These studies did not concern poultry production and their results were sometimes specific to a particular type of animal production, such as fish farming [32], where a significant farm effect was observed. In the study of antimicrobial consumption in pigs, the factors identified were related to the specific pathogen free status of pig herds, room design and pig bodyweight [23]. The results obtained in the present study tend to confirm the feasibility of the approach adopted in a preliminary trial in determining those factors likely to influence antimicrobial consumption. Since 2001, some changes have occurred in turkey broiler production in France: the consequences of the foodstuff modification (suppression of growth promoters and meat and bone meals) are better controlled and considerable efforts have been put on a large information of farmers about good farming practices and on a systematic implementation of biosecurity rules. It would therefore be interesting to determine whether these changes have been associated with a corresponding reduction in antimicrobial use.

\section{ACKNOWLEDGEMENTS}

The authors are grateful to the farmers, the investigators Mrs Bourdette, Cnapelynck, Palussiere and Messrs Berthelot, Prigent, Roy, for their active cooperation in this study, Mrs Conan, End, Müller, Messrs Delabrosse and Nicolas for their collaboration and to Mrs Guittet and $\mathrm{Mr}$ Eterradossi for their judicious advice about the manuscript. Data was collected in collaboration with the Chambres d'Agriculture de Bretagne, des Pays de Loire et du Centre, with financial support from the Office National Interprofessionnel des Viandes de l'élevage et de l'aviculture, the Conseil Régional de Bretagne and the Conseil Régional des Pays de la Loire, the Food Safety Directorate of the French Ministry of Agriculture, Food, Fisheries and Rural Affairs. This study is part of a research project on the pharmaco-epidemiology of veterinary antimicrobials funded by the Food Safety Directorate of the French Ministry of Agriculture, Food, Fisheries and Rural Affairs.

\section{REFERENCES}

[1] Allison P.D., Logistic regression Using SAS System: Theory and application, SAS Institute Inc., Cary, NC, 1999, pp. 133-148.

[2] Anonymous, Résultats de l'enquête 19992000, Chambre d'Agriculture du Morbihan avec la participation des Chambres d'Agriculture du Grand Ouest, 2000.

[3] Anonymous, Dictionnaire des médicaments vétérinaires, $10^{\mathrm{e}}$ édition, Éditions du Point Vétérinaire, Maisons-Alfort, France, 2001. 
[4] Anonymous, La dinde par les chiffres, Comité interprofessionnel de la dinde française, Mordelles, France, 2002.

[5] Anonymous, DANMAP 2001 - Use of antimicrobials agents and occurrence of antimicrobial resistance in bacteria from food animals, foods and humans in Denmark, Bager F., Emborg H.D., Heuer O.E. (Eds.), Danish Veterinary Institute, Copenhagen, Denmark, 2002, 69 p.

[6] Bager F., Jensen V.F., Jacobsen E., Surveillance experiences from DANMAP and VetStat - usage of antimicrobials in animals, DIAS Report Animal Husbandry 57 (2004) 61-66.

[7] Britten N., Ukoumunne O., The influence of patient's hopes of receiving a prescription on doctor's perceptions and the decision to prescribe: a questionnaire survey, Br. Med. J. 315 (1997) 1506-1510.

[8] Carey V.J., Zeger S.L., Diggle P., Modelling multivariable binary data with alternating logistic regressions, Biometrika 80 (1993) 517-526.

[9] Chauvin C., Madec F., Guillemot D., Sanders $\mathrm{P}$., The crucial question of standardisation when measuring drug consumption, Vet. Res. 32 (2001) 533-543.

[10] Chauvin C., Belœil P.A., Orand J.P., Sanders P., Madec F., A survey of group-level antibiotic prescriptions in pig production in France, Prev. Vet. Med. 55 (2002) 109-120.

[11] Chauvin C., Hardy A., Le Bouquin S., Orand J.P., Première base de données vétérinaires française - le projet O.A.C.A. en filière volailles, in: Second colloque de pharmacoépidémiologie, Paris, France, 22-23 octobre 2002.

[12] Dewey C.E., Cox B.D., Straw B.E., Bush E.J., Hurd H.S., Associations between off-label feed additives and farm size, veterinary consultant use and animal age, Prev. Vet. Med. 31 (1997) 133-146.

[13] Grave K., Nilsson L., Greko C., Mørk T., Odensvik O., Rønning M., The usage in Norway and Sweden of veterinary antibacterial drugs for mastitis during 1990-1997, Prev. Vet. Med. 42 (1999) 45-55.

[14] Grave K., Kaldhusdal M., Kruse H., Fevang Harr L.M., Flatlandsmo K., What happened in Norway after the ban of avoparcin? Consumption of antimicrobials by poultry, Prev. Vet. Med. 62 (2004) 59-72.

[15] Heier B.T., Hogasen H.R., Jarp J., Factors associated with mortality in Norwegian broiler flocks, Prev. Vet. Med. 53 (1999) 147_ 158.

[16] Jensen V.F., Jacobsen E., Bager F., Veterinary antimicrobial-usage statistics based on standardized measures of dosage, Prev. Vet. Med. 64 (2004) 201-215.

[17] Kaneene J.B., Miller R., Description and evaluation of the influence of veterinary presence on the use of antibiotics and sulfonamides in dairy herds, J. Am. Vet. Med. Assoc. 201 (1992) 68-76.

[18] Katz J., Carey V.J., Zeger S.L., Sommer A., Estimation of design effects and diarrhea clustering within households and villages, Am. J. Epidemiol. 138 (1993) 994-1006.

[19] Larsen P.B., Consequences of termination of AGP use for pig health and usage of antimicrobials for therapy and prophylaxis, DIAS Report Animal Husbandry 57 (2004) 67-71.

[20] Lunde P.K.M., Baksaas I., Halvorsen T., Stromnes B., Oydvin K., The methodology of drug utilization studies, in: Bergman U., Grimson A., Welsterholm B. (Eds.), Studies in drug utilization, WHO Reg. Publ., Eur. Ser. 8 (1979) 17-28.

[21] Mangione-Smith R., McGlynn E.A., Elliott M.N., Krogstad P., Brook R.H., The relationship between perceived parental expectations and paediatrician antimicrobial prescribing behaviour, Pediatrics 103 (1999) 711-718.

[22] Nicholls T., Acar J., Anthony F., Franklin A., Gupta R., Tamura Y., Thompson S., Threlfall E.J., Vose D., van Vuuren M., White D.G., Wegener H.C., Costarrica M.L., Office International des Epizooties Ad hoc Group, Antimicrobial resistance: monitoring the quantities of antimicrobials used in animal husbandry, Rev. Sci. Tech. Off. Int. Epizoot. 20 (2001) 841-847.

[23] Nielsen E.O., Hassing A.G., Wachmann H., Baekbo P., Petersen H.H., Nielsen J.P., Use of antibiotics in Danish pork production: risk factors associated with high frequency of treatment, Dansk Vet. Tidsskr. 85 (2002) 613 (in Danish).

[24] Olivier B., Bouvarel I., Berthelot A., Health management practices in turkey broiler farms, Sci. Tech. Avi. 40 (2002) 21-28 (in French).

[25] Patterson J.A., Burkholder K.M., Application of prebiotics and probiotics in poultry production, Poult. Sci. 82 (2003) 627-631.

[26] Perez J.M., Bories G., Aumaitre A., BarrierGuillot B., Delaveau A., Gueguen L., Larbier M., Sauvant D., Consequences of the replacement of meat and bone meals and fats of animal origin on farm animals and for human consumers, Prod. Anim. 15 (2002) 87-96. 
[27] Petronis K.R., Anthony J.C., A different kind of contextual effect: geographical clustering of cocaine incidence in the USA, J. Epidemiol. Community Health 57 (2003) 893-900.

[28] Petronis K.R., Anthony J.C., Social epidemiology, intra-neighbourhood correlation, and generalised estimating equations, J. Epidemiol. Community Health 57 (2003) 914.

[29] Preisser J.S., Arcury T.A., Quandt S.A., Detecting patterns of occupational illness clustering with alternating logistic regressions applied to longitudinal data, Am. J. Epidemiol. 158 (2003) 495-501.

[30] Tablante N.L., Brunet P.Y., Odor E.M., Salem M., Herter-Dennis J.M., Hueston W.D., Risk factors associated with early respiratory disease complex in broiler chickens, Avian Dis. 43 (2002) 424-428.

[31] Te Winkel G.P., Biosecurity in poultry production: where are we and where do we go? Acta Vet. Hung. 45 (1997) 361-372.

[32] Thorburn M.A., Teare G.F., Martin S.W., Moccia R.D., Group-level factors associated with chemotherapeutic treatment regimens in land-based trout farms in Ontario, Canada, Prev. Vet. Med. 50 (2001) 165-176.

[33] Tornoe N., Consequences of termination of AGP use for broiler health and usage of antimicrobials for therapy and prophylaxis, DIAS Report Animal Husbandry (2004) 73-76. 